\title{
Aprendizaje, enseñanza, conocimiento, tres acepciones del constructivismo
}

\author{
Implicaciones para la docencia
}

Felipe Martínez Rizo*

Muchos docentes dicen identificarse con el constructivismo, pero este término se usa en al menos tres sentidos diferentes, dependiendo de si se refiere a la noción del aprendizaje, la enseñanza o el conocimiento. El artículo pretende mostrar que la solidez de las tres acepciones es diferente, y trata con más amplitud la acepción relativa a enseñanza. Se identifican implicaciones de lo anterior para el trabajo docente, a partir de estudios empíricos sobre prácticas en aula que llevan a cuestionar la supuesta adhesión de muchos docentes a los principios constructivistas y se señalan aspectos positivos. En la conclusión se busca derivar reflexiones sobre las políticas que llevarían a prácticas apegadas al constructivismo en sus acepciones más sólidas y que pudieran llevar a una mejora sustancial de los aprendizajes. Esas políticas deberían privilegiar la mejora de la formación y actualización de los docentes.

Many teachers declare themselves as constructivists, however this term is used in at least three different senses, depending on whether it refers to the notion of learning, teaching, or knowledge. The present article aims to show the differences between the tree meanings and deals more broadly with the meaning associated to the notion of teaching. Based on a series of empirical studies on classroom practices that lead to questioning the supposed adherence of many teachers to constructivist principles, we identified several implications of the afore mentioned misconception on teaching practices. We also point out some positive aspects. In our conclusions we aim to promote the reflection regarding the policies needed to develop actually constructivist teaching practices which might, in turn, result in a substantial improvement in learning. These policies should give priority to improving the training and updating of teachers.
Palabras clave

Aprendizaje

Enseñanza

Conocimiento

Constructivismo

Conductismo

Docencia

Keywords

Learning

Teaching

Knowledge

Constructivism

Behaviorism

Recepción: 20 de octubre de 2020 | Aceptación: 2 de abril de 2021 DOI: https://doi.org/10.22201/iisue.24486167e.2021.174.60208

* Investigador honorario del Departamento de Educación de la Universidad Autónoma de Aguascalientes (México). Temas de investigación: calidad y evaluación de la educación; estudio de la práctica docente; enseñanza de ciencias naturales. Publicaciones recientes: (2020), El nuevo oficio del investigador educativo. Una introducción metodológica, México/Aguascalientes, COMIE/UAA; (2020, coord.), La autonomía en la coyuntura actual, Aguascalientes, Universidad Autónoma de Aguascalientes. CE: felipemartinez.rizo@gmail.com 
INTRODUCCIÓN:

\section{PROPÓSITO DEL ARTÍCULO}

El constructivismo es la postura pedagógica que casi todos los docentes dicen seguir, aunque la observación del aula muchas veces lleva a otra conclusión. Este artículo muestra que el término constructivismo se usa en tres sentidos distintos, que se refieren a la manera de concebir el aprendizaje, la enseñanza y el conocimiento. La primera acepción se sitúa en el ámbito de la psicología y la pedagogía y se refiere a procesos del estudiante; la segunda se refiere a lo que hace el docente, y pertenece, por ende, a la didáctica (didáskalos, maestro en griego); y la tercera nos remite al terreno de la filosofía de la ciencia, la epistemología.

El artículo pretende también mostrar que la solidez de las tres acepciones es diferente, $y$ discutir algunas consecuencias para la práctica docente. En un texto de extensión limitada es imposible dar cuenta con un mínimo detalle de autores y corrientes relevantes de las tres acepciones del constructivismo consideradas, en especial de las teorías del aprendizaje relativas a la primera; ello hace que el propósito se limite a cuestionar las visiones dicotómicas simplistas que todavía prevalecen en un número considerable de docentes de educación básica.

\section{EL APRENDIZAJE: PRIMERA}

\section{ACEPCIÓN DEL CONSTRUCTIVISMO}

Las raíces de las actuales ideas sobre el aprendizaje se remontan a las concepciones idealistas y empiristas del conocimiento en la filosofía griega; durante siglos prevaleció la visión de Platón, que minimizaba el papel de los sentidos en el avance del conocimiento, frente a la postura de Aristóteles, lo que en los siglos XVI y XVII cambió con Francis Bacon y John Locke, quienes sostuvieron que la fuente de todo conocimiento no es otra que las percepciones de la realidad externa que llegan a la mente por los sentidos.

Por la misma época el empirismo comenzó a influir en la pedagogía con Comenio (Jan
Amos Komensky), quien adoptó la postura de Bacon de que nada entra en el intelecto si no es por los sentidos, y la transformó en principio pedagógico, al postular que, para que el niño desarrolle un concepto, hay que partir de la observación de la realidad o de una imagen e ir al concepto por la palabra que lo representa, es decir, ir de la experiencia al lenguaje, en cuatro etapas: el lenguaje balbuceante, que se aprende en el regazo materno; el correcto, que se enseña en la escuela pública en lengua vernácula; el lenguaje elegante del gimnasio, escuela de gramática latina; y el claro y convincente que se desarrolla en la universidad y gracias a los viajes.

En el siglo XVIII Immanuel Kant puso en cuestión tanto el empirismo de Bacon y Locke como el idealismo platónico, con la idea de que el conocimiento implica la percepción de una realidad externa mediante los sentidos, pero también la sistematización de esas experiencias, para lo que inevitablemente debemos aplicar las categorías mentales presentes en nuestro aparato cognitivo. En los siglos XVIII y XIX la filosofía siguió influyendo en las ideas educativas con Pestalozzi, que aceptó el empirismo baconiano, con la corrección de Kant de que nuestra aprehensión del mundo no se reduce a las sensaciones, sino que es más holística.

El desarrollo de la psicología y otras ciencias del ser humano desde fines del siglo XIX hizo avanzar las ideas sobre el aprendizaje y dio lugar a corrientes que se suelen organizar en dos grupos: conductistas y constructivistas. Dentro de cada uno de estos conjuntos hay diferencias considerables y también hay posturas que escapan a esa dicotomía, como advertía ya hace más de 40 años un difundido manual sobre teorías del aprendizaje (Swenson, 1980).

El autor clasifica las corrientes de la primera mitad del siglo XX en tres grupos: teorías de la contigüidad, con el condicionamiento clásico de Pavlov y el asociacionismo de Watson y Guthrie; teorías del reforzamiento de Thorndike, seguido por Hull y luego Skinner; y teorías cognitivas, con Tolman y la corriente de 
la Gestalt, que buscaban superar los limites de las teorías anteriores, que explicaban aprendizajes simples, pero no podían dar cuenta de los más complejos. Swenson señala que algunos conductistas, incluso Skinner, adoptaron conceptos de los cognitivistas. Identifica también autores eclécticos (Swenson, 1980).

La segunda parte de la obra presenta corrientes "recientes": avances de las ideas de Pavlov y de Skinner, con la teoría del aprendizaje social de Bandura, y luego posturas cognitivistas, con Piaget y la teoría del procesamiento de la información de Norman, e incluso presenta trabajos tempranos de neurofisiología del aprendizaje como los de Pribram (Swenson, 1980).

Swenson reconoce que, en la década de 1970, cuando preparaba su obra, el autor dominante era sin duda Skinner, y las ideas de Piaget sólo comenzaban a ser conocidas en Estados Unidos. Por ello su presentación de teorías "recientes" dista mucho de ser actual; menciona a Piaget, pero no llegó a cubrir otros autores fundamentales de las posturas cognitivistas, como Vygotsky o Bruner. Pese a ello, desde entonces apunta que, si bien los autores que cita "no han resuelto del todo las grandes cuestiones relativas al aprendizaje, sí han generado investigación que ha llevado a la eliminación de muchos dogmas simplistas" (Swenson, 1980: 185).

Entre los avances que Swenson no cubre conviene mencionar, en primer lugar, desarrollos del conductismo, con Carroll o Bloom, con la propuesta de aprendizaje de dominio (mastery learning) que introduce la idea de que la capacidad de aprender no es exclusiva de una minoría, sino que todos los niños pueden aprender, pero a distinto ritmo (Crahay, 2002).

Una visión más actual del tema muestra que las posturas cognitivistas del aprendizaje, a las que alude el término constructivismo en una primera acepción, se gestaron desde la primera mitad del siglo XX y se manifestaron con fuerza a partir de la década de 1960; estas posturas enfrentaron al conductismo desde el constructivismo de enfoque personal con Piaget, y el que destaca la importancia del contexto social, con Vygotsky. En este grupo se puede identificar, además, el constructivismo cultural de Bruner (Crahay, 2002).

Otras revisiones de teorías del aprendizaje reafirman la idea de que algunas no encajan en la dicotomía conductismo-constructivismo, pues retoman elementos de la revolución cognitiva y del conductismo: el cognitivismo social de Bandura y la teoría de los niveles de aprendizaje de Gagné; la del procesamiento de información; la del aprendizaje situado de Ausubel; o la del proceso por el que un novato se vuelve experto (Abell et al., 2010; Knoers, 1994; Mayer, 2014; Schunk, 2014).

En realidad, las posturas constructivistas no descartan en forma absoluta las aportaciones del conductismo, sino que las desarrollan y enriquecen, pero una visión simplista lleva a creer que en el conductismo el aprendizaje simplemente es fruto de una acción externa que afecta a sujetos pasivos. Esto tiene algún fundamento, en tanto en el conductismo la atención se centra en los procesos de enseñanza y se destaca el papel activo del maestro, con lo que los procesos de aprendizaje y los alumnos quedan en un segundo plano, con un papel aparentemente pasivo; pero lo que dice realmente el conductismo es que no se puede saber qué pasa en la mente de las personas, y lo único observable son las conductas que llevan a cabo, las cuales, desde luego, resultan de lo que pasa en la mente, pero en una forma que escapa a nuestro conocimiento. La enseñanza de docentes, y el aprendizaje de alumnos tienden a verse en términos de trasmisión y recepción, con los maestros en el papel principal, activo, y los alumnos-aprendices en un rol subordinado. Sin embargo, como muestra el caso de Bloom, en el conductismo el papel del estudiante no es necesariamente pasivo; aunque parezca no hacer nada por estar inmóvil, el aprendiz puede estar muy activo si presta atención en forma concentrada y por largo tiempo a lo que dice el maestro. 
La trasmisión de un conocimiento es algo activo, pero la recepción no es algo pasivo, como sugiere la metáfora de la "educación bancaria" de Freire, que asimila al aprendiz a una alcancía en la que el maestro deposita monedas de conocimiento. La metáfora, que se quiere positiva, del niño como esponja que absorbe todo lo que ve o escucha a su alrededor, tampoco hace justicia al esfuerzo que hace un estudiante que sigue atentamente las explicaciones del maestro.

Con este matiz la visión conductista del aprendizaje sí minimiza el papel activo del aprendiz, lo que cambió el constructivismo al redefinir la función del docente, pues "enseñar no es trasmitir el saber... ni ejercer facultades o potencialidades latentes. Es organizar experiencias que inducen al educando a reestructurar o remodelar su saber intuitivo" (Crahay, 2002: 248).

Las corrientes constructivistas tienen en común ver el aprendizaje como producto de sujetos activos que desarrollan conocimientos mediante procesos de construcción complejos, ya que sus mentes no están en blanco (tabula rasa), sino que tienen ideas adquiridas previamente; por ello construir otras implica sustituir las previas o modificarlas incorporando nuevos elementos. La revolución cognitiva da lugar al constructivismo, en una primera acepción del término.

El cambio fundamental de la revolución cognitiva consiste en afirmar que sí es posible conocer lo que pasa en la mente de las personas, no sólo mediante inferencias a partir de las conductas observables, sino gracias a la información que pueden dar los mismos sujetos, con las debidas precauciones, y con técnicas como las entrevistas clínicas piagetianas y las diversas formas de entrevistas cognitivas. Gracias a estas técnicas se pueden explorar los procesos por los que los aprendices construyen conocimientos a partir de lo que observan y lo que escuchan, lo cual confrontan con las ideas que ya tienen para complementarlas, precisarlas o modificarlas.
Frente al conductismo que, en la versión simplificada extendida entre maestros, propondría un rol pasivo del aprendiz y uno activo del docente, reducido a exponer y explicar (trasmitir) información que el alumno sólo debe recordar; para el constructivismo el aprendizaje depende directa e inmediatamente de lo que hace el aprendiz, y no de lo que hace el docente; las acciones de éste pueden ser decisivas, pero de manera indirecta y mediata, en la medida en que logran que los alumnos lleven a cabo acciones que propicien el desarrollo de los aprendizajes que se quiere promover.

El docente ya no debe limitarse a presentar, explicar y demostrar, sino que debe buscar formas de llevar a los alumnos aprendices a realizar actividades mentales por medio de las cuales construyan conocimientos nuevos, a partir de los que ya tenían y de los elementos adicionales que aporta el docente, pero también de su propia observación de la realidad, y de la indagación activa de fuentes de información diferentes al maestro. La intervención del docente tomará frecuentemente la forma de preguntas abiertas que hagan pensar a los alumnos, cuestionamientos que los hagan poner en tela de juicio sus ideas previas, orientaciones sobre qué observar, etc.

Lo anterior se refiere a aspectos cognitivos, pero también importan los actitudinales y afectivos: si un alumno no quiere aprender algo, simplemente no lo hará, entendiendo que no se trata de deseos del momento, sino de aquéllos que surgen de un interés profundo; adicionalmente, la disposición favorable a aprender se dará si no lo ve como amenazante o inaccesible, sino que lo encuentra atractivo y a su alcance, lo que a su vez supone confianza en sus posibilidades y autoestima. Esto lleva a las teorías de la motivación y sus implicaciones para el aprendizaje, no sólo de aquéllas desarrolladas por autores llamados humanistas, sino también en el ámbito de conductismos y constructivismos.

Lo que hace el docente es indisociable de lo que hacen los alumnos, y si el fin de todo proceso educativo es conseguir que los alumnos 
aprendan, los aprendizajes dan sentido a lo que pasa en el aula. Por ello toda teoría de la enseñanza supone cierta teoría del aprendizaje.

\section{LA ENSEÑANZA: SEGUNDA ACEPCIÓN DEL CONSTRUCTIVISMO}

Como se ha apuntado ya, cada familia de teorías del aprendizaje tiene una concepción de la enseñanza. Si el aprendizaje se concibe como conexión o fortalecimiento de ciertas respuestas, la enseñanza consistirá en ejercicio y práctica (drill \& practice) o en aplicar refuerzos en el momento adecuado; si se ve como adquisición de información se prestará atención al papel de la memoria de trabajo de corto plazo, y en la de largo plazo en el proceso de aprender y recordar; y si se entiende como construcción de conocimientos por parte de los aprendices, no consistirá centralmente en exposiciones o demostraciones que hace el docente y los alumnos aprenden, en un sentido que puede reducirse a la simple memorización, pero debe incluir la comprensión.

Hay que subrayar que posturas conductistas avanzadas sobre la enseñanza tienen elementos congruentes con el cognitivismo. Un ejemplo es la aportación de Benjamín Bloom a la noción de evaluación formativa. Desde 1968 Bloom señalaba que lo esencial de dicha evaluación es la retroalimentación y precisaba que no sólo debe dirigirse a los alumnos, sino también a los maestros. La evaluación no debe ser el punto final del proceso docente, sino algo permanente, dimensión esencial de una enseñanza de buena calidad e indisociable de ella, pues posibilita que un docente corrija su forma de enseñar y la mejore. Bloom precisa que esto:

...no es simplemente volver a enseñar, lo que típicamente quiere decir repetir las explicaciones originales en voz más alta y más despacio; implica la utilización de enfoques que adapten la enseñanza a las diferencias de estilos de aprendizaje, modalidades y formas de inteligencia de los alumnos (Guskey, 2007: 66-67).
La enseñanza que pone al alumno en un papel subordinado y al docente en el protagónico se conoce como enseñanza frontal. El docente está a cargo de desarrollar los temas o hacer que los estudiantes lean pasajes del libro de texto $\mathrm{u}$ otros materiales buscando que aprendan (recuerden, memoricen) términos, conceptos, datos, fórmulas, nombres y fechas, lo que se verifica con evaluaciones que consisten en preguntas de respuesta corta o de selección de respuesta.

La perspectiva constructivista asigna al alumno el papel principal, lo que se puede entender de dos maneras: una en que el docente sigue siendo importante para ayudar a cada alumno a transitar de sus ideas previas a las que se pretende debe desarrollar, a sabiendas de que el paso no es fácil de dar y muchos estudiantes no lo conseguirán sin ayuda; la otra forma considera que el rol del docente debería reducirse al mínimo, y asegurar solamente un aula donde haya el ambiente y las condiciones para que la creatividad de los alumnos se manifieste.

Es esta una segunda acepción del constructivismo: si el aprendizaje depende ante todo del aprendiz, la mejor forma de enseñar será por descubrimiento, con mínima intervención del maestro y aprendices activos y creativos. En la primera acepción el constructivismo es muy aceptado; en la segunda no. Un autor crítico de esa enseñanza llamada constructivista dice:

Muchos aspectos del constructivismo son incuestionables. Por ejemplo, es claro que todos construimos representaciones mentales del mundo externo que podemos usar para funcionar en él. En ese sentido, todo aprendizaje es básicamente constructivista y no sé de ningún teórico que objete esta manera de caracterizar el aprendizaje. Pero una docencia constructivista, dirigida a enseñar a las personas cómo construir conocimiento sin dar información a los aprendices es algo totalmente diferente (Sweller, 2009: 127).

Según este autor, la segunda manera de concebir la enseñanza comienza con Bruner, 
inspirado en Piaget. Sweller retoma la distinción, en perspectiva biológica, entre conocimiento primario - para el que nuestro cerebro está preparado (hard-wired) gracias a milenios de evolución- y conocimiento secundario, de épocas más recientes. El aprendizaje de la lengua materna es ejemplo de los primeros; el de la lectoescritura lo es de los segundos. Aunque la lectoescritura también se basa en el aprendizaje primario de la lengua oral, no evolucionamos para aprender a leer $y$ escribir, para lograrlo necesitamos de alguien que nos enseñe (Sweller, 2009).

Conviene aclarar que, desde el primer texto en que Bruner (1961) propone el aprendizaje por descubrimiento, su postura no es de completa ausencia de apoyo por parte del maestro, lo que precisó poco después, en una reunión organizada en 1964 por el Social Science Research Council, en la que una docena de estudiosos discutió críticamente la noción de aprendizaje por descubrimiento (cfr. Shulman y Keislar, 1966). Bruner precisó sus ideas como sigue:

Parece muy improbable esperar que cada organismo redescubra la totalidad de su cultura... es igualmente improbable que el largo periodo de dependencia propio de nuestra especie haya sido diseñado para la técnica más ineficiente posible para volver a encontrar lo conseguido a lo largo de mucho tiempo, o sea por descubrimiento... en el ámbito cultural, la primera forma de aprendizaje, esencial para que la persona se vuelva humana, no es el descubrimiento, sino el tener un modelo. El disponer permanentemente de un modelo, la respuesta constante a cada individuo, respuesta tras respuesta, el ir y venir entre dos personas, eso constituye el aprendizaje por descubrimiento guiado por un modelo accesible (Bruner, 1966: 102).

Hay, pues, dos formas de entender el aprendizaje por descubrimiento que no suelen tener en cuenta las discusiones al respecto, todavía frecuentes: una notable inició con un artículo de Kirschner et al. (2006), seguida por un debate en la American Educational Research Association (AERA 2007) y el libro ya citado de Tobias y Duffy (2009): unos capítulos de esta obra defienden la enseñanza constructivista e incluyen, con matices, varias formas de guía por parte del maestro; otros capítulos critican la postura constructivista, y otros abordan campos específicos. La lectura de esos textos muestra que las posturas contrastadas no están tan lejos entre sí como se piensa, y nadie pone en duda la solidez de la visión constructivista del aprendizaje. Uno de los autores dice:

Ha habido una tendencia... a identificar constructivismo con enseñanza mínimamente guiada. Esto es muy general, pero oscurece otro sentido del término constructivismo: que el aprendizaje es un proceso activo, que el conocimiento se construye. Este es un punto muy importante sobre el que hay un gran acuerdo en la literatura. Al rechazar el "constructivismo" no queremos regresar a una visión del aprendizaje como adquisición pasiva del conocimiento (Kintsch, 2009: 223).

Otros autores, retomando a Bruner, advierten que la forma en que sus críticos entienden la enseñanza constructivista:

Parece derivarse de una idea equivocada muy extendida: que ese enfoque implica mandar a los niños a un terreno, armados con una pala y una lupa, con la esperanza de que ellos "redescubrirán” principios científicos básicos. Sostenemos que esto no es un ejemplo de aprendizaje por descubrimiento, sino de mala enseñanza. Bruner tenía muy claro... que el aprendizaje por descubrimiento... implica preparación cuidadosa, orientación y modelamiento por parte del maestro. Por ello, referirse a tales enfoques como "no guiados" o incluso "mínimamente guiados" es una burda deformación (Sommerfeld y Lester, 2009: 266-267). 
Las dos formas de entender la segunda acepción del constructivismo son:

- Descubrimiento puro (pure discovery): la intervención docente reducida al mínimo; lo ideal, dejar que los niños, por sí mismos y sin ayuda, exploren, indaguen, manipulen objetos, busquen formas de resolver problemas, etc., sea individualmente, sea en grupo.

- Descubrimiento guiado (guided discovery): reconoce la importancia del papel del aprendiz; se destaca el rol indispensable del docente para apoyar el trabajo del alumno, llamar su atención sobre puntos importantes, reorientarlo si es necesario, identificar obstáculos y ayudar a superarlos. Un niño puede estar activo conductual o cognitivamente (behavioral vs cognitive activity), y lo importante es la actividad cognitiva, acompañada o no de actividad conductual.

Estas dos visiones de la enseñanza como aprendizaje por descubrimiento en ciencias tienen equivalentes en otras áreas. Partidarios de cada visión se enfrentan en polémicas como las de quienes prefieren una enseñanza sistemática de la lectoescritura y los que plantean que la mejor forma de que un niño aprenda a leer es exponerlo tempranamente a material atractivo y dejarlo avanzar solo (lenguaje total, whole language). Las guerras de matemáticas son otro ejemplo. Las polémicas persisten, aunque la investigación ya permite valorar la efectividad de las dos formas de entender la enseñanza, y muestra que no hay sustento para posturas extremas, que combinar dirección por parte del maestro y exploración por el alumno es lo más adecuado.

Richard Mayer, con base en una revisión de investigaciones desde 1950, comparó el potencial de métodos de enseñanza basados en descubrimiento puro o guiado, considerando también la distinción entre actividad manual y mental, y concluyó:
Mi revisión de literatura... no ofrece apoyo para métodos de descubrimiento puro. ¿Quiere decir esto que el constructivismo está equivocado? Sin duda quiere decir que un enfoque doctrinario no lleva a una buena práctica educativa... la fórmula "constructivismo=niños conductualmente activos" lleva a un desastre. Sin embargo, el fracaso del enfoque de descubrimiento puro como método de enseñanza no implica forzosamente que el constructivismo sea una teoría equivocada o la actividad conductual un método de instrucción equivocado... El descubrimiento guiado parece ofrecer el mejor método para promover un aprendizaje constructivista. El reto que plantea una enseñanza basada en el descubrimiento guiado es el de saber cuánta guía y de qué tipo ofrecer... La actividad puede ayudar a promover aprendizaje significativo, pero en vez de actividad conductual per se (manipulación, discusión, exploración libre) la actividad que sí promueve el aprendizaje significativo es la cognitiva (p. ej. seleccionar, organizar e integrar conocimientos). En vez de consistir sólo en aprender haciendo o discutiendo, el acercamiento más auténtico a un aprendizaje constructivista es aprender pensando. Los métodos que se basan en hacer o discutir deben valorarse no por cuánta acción o discusión está implicada, sino más bien por el grado en que promueven un procesamiento cognitivo apropiado (Mayer, 2004: 17).

Estas precisiones importan porque la influencia del constructivismo "va mucho más allá de las revistas de investigación... en muchos países... se ha adoptado como la teoría pedagógica oficial... es ubicuo en los programas de formación de maestros en América Latina”. La influencia del constructivismo radical parece disminuir y dejar sitio a "principios más realistas, limitados, y filosófica y educativamente mejor fundamentados" (Matthews, 2017: 388).

En apoyo a que la investigación cuestiona la eficacia de la pedagogía mínimamente 
guiada, Matthews menciona las guerras de lectura, que enfrentan a partidarios de lenguaje total y enseñanza fonética, y señala que, pese a la solidez de estudios que muestran que las estrategias de lenguaje total no funcionan, se ha frustrado "la adquisición de cultura escrita de miles, si no es que millones de niños". Lo mismo pasa con las guerras de matemáticas (Matthews, 2017: 410).

Los últimos 50 años de investigación sobre este tema han recabado evidencias avasalladoras e inequívocas de que el aprendizaje no guiado o mínimamente guiado es claramente menos efectivo y eficiente que la asesoría diseñada especialmente para apoyar el procesamiento cognitivo necesario para aprender. El aprendizaje mínimamente guiado no sólo resulta inútil para la mayor parte de los alumnos; incluso puede resultar perjudicial para algunos... no acentúa el logro estudiantil, del mismo modo que arrojar a alguien que no sabe nadar de un bote a la mitad de un lago no ayuda a aprender a nadar (Kirschner et al., 2006: 75).

Si se rechaza la versión radical de la enseñanza mínimamente guiada sin descartar la idea de que el aprendiz es un sujeto activo, entonces el reto real es el de "saber cuánta guía y de qué tipo ofrecer" (Clark, 2009; Mayer, 2009), y de eso es de lo que tratan los esfuerzos por renovar la enseñanza en matemáticas y ciencias. En esa dirección apunta la distinción de Shulman entre conocimiento de contenidos y conocimiento pedagógico de contenidos (CPC, pedagogical content knowledge, PCK): además de dominar lo que el alumno debe aprender, el docente necesita otro conocimiento, el que específicamente se requiere para la tarea de enseñar:

Un segundo tipo de conocimiento de contenido es conocimiento pedagógico que, más allá del dominio del contenido per se, llega a lo que se requiere para enseñar... una forma particular que incorpora los aspectos del contenido más afines a su carácter de enseñable... Incluyo las formas más útiles de representar temas que se enseñan: analogías, ilustraciones, ejemplos, explicaciones y [las] demostraciones más poderosas; en una palabra, formas de representar y formular contenido que lo hacen comprensible a otros... incluye también una comprensión de lo que hace fácil o difícil el aprendizaje de tópicos particulares: las ideas previas que los alumnos de distintas edades y procedencias traen consigo... y si esas ideas previas son erróneas, como ocurre muchas veces, los maestros necesitan un conocimiento de las estrategias potencialmente más fructíferas para reorganizar la comprensión de los aprendices... (Shulman, 1986: 9-10).

Sobre ideas equivocadas de los niños, Shulman cita especialistas en enseñanza de matemáticas que aconsejan no preguntar por qué uno hizo algo tonto al cometer un error, sino ponerse en su lugar y preguntarse qué es lo que hace que el niño encuentre eso razonable. Al reconocer la dificultad de ese conocimiento, subraya la complejidad de la docencia citando a Aristóteles:

Enseñar es la forma más elevada de comprensión; ninguna prueba sobre la comprensión humana es más exigente que ver si uno puede tomar algo que cree que conoce, y logra enseñarlo a alguien más (Shulman, 1990: 401).

Ball y Hill concretan la noción de CPC en enseñanza de matemáticas con la expresión mathematical knowledge for teaching (MKT), que describen así:

El conocimiento necesario para enseñar a multiplicar implica, más que ser capaz de hacer esos cálculos, dominar las matemáticas de $4^{\circ}$ de primaria y poco más. Es una mezcla compleja de conocimientos, habilidades, procesos de razonamiento y otros hábitos mentales, como capacidad de ver la perspectiva de otra persona $y$ entender lo que hace, lo que necesita un 
maestro, y no un matemático o físico (Ball y Hill, 2009: 88).

Detectar sutiles diferencias al elegir ejemplos a utilizar es tarea que implica una habilidad matemática crítica para la docencia, que personas de buena formación matemática o investigadores no tienen. La enseñanza requiere una peculiar manera de ver los contenidos, que no es común en los que usan la matemática para otros propósitos (Ball y Hill, 2009: 90).

\section{La trasposición didáctica de Chevalard y} sus ingenierías para enseñar ideas matemáticas tiene paralelismo con el conocimiento pedagógico de contenido de Shulman. Los avances de la didáctica en Francia, Alemania, Rusia y otros países coinciden con los del ámbito anglosajón. La trasposición didáctica "refleja una separación entre saber sabio y enseñado, se refiere a la forma en que los contenidos a enseñar se adaptan y remodelan", y corresponde al contraste anglosajón entre contenido y su aplicación en enseñanza, y entre conocimiento del contenido y conocimiento pedagógico del contenido (Alexander, 2000: 543). En todos los casos se trata del paso de una forma de saber complejo a una más accesible al alumno.

\section{EL CONOCIMIENTO: TERCERA ACEPCIÓN DEL CONSTRUCTIVISMO}

Esta acepción se sitúa en el ámbito de la epistemología, como una forma de entender el conocimiento, tanto en general, como el propio de las ciencias. Al respecto hay dos posturas extremas: una, ingenuamente optimista, ve a la ciencia como conocimiento perfecto, totalmente superior al conocimiento no científico; otra, radicalmente negativa, según la cual los conocimientos científicos no son superiores a los de sentido común: todo conocimiento es una construcción social, y no es posible detectar superioridad alguna del conocimiento científico. Esta postura se llama también constructivismo, en una tercera acepción del término.
La visión ingenuamente positiva de la ciencia se relaciona con el positivismo, que se remonta a la obra de Augusto Comte en 18301840. A fines del siglo XIX y en la década de 1920 se desarrolló el neo-positivismo, que desde mediados del siglo XX fue abandonado por los especialistas, dejando el lugar a varios postpositivismos. Entonces cobró fuerza la visión radicalmente negativa del construccionismo epistemológico, que se ha llamado también posmoderna y deconstruccionista.

Las posturas extremas se encuentran entre maestros e investigadores educativos y sociales, en versiones que muestran desconocimiento de sus alcances y límites, así como de las ideas matizadas que sostienen hoy los especialistas. Estas ideas implican reconocer la existencia del mundo como realidad externa al sujeto cognoscente, y aceptar que nuestro conocimiento es imperfecto, marcado por nuestras categorías, como apuntó Kant, y como desde mediados del siglo XX coinciden en plantear post-positivistas como Popper $y$ neo-hermenéuticos como Gadamer.

Frente a las dos posturas extremas sobre los conocimientos producidos por las ciencias, la epistemología actual ofrece una visión matizada, según la cual:

- Los conocimientos científicos, sin ser perfectos ni acabados, son más precisos y completos que los de sentido común o los pseudocientíficos.

- Los científicos no son personas especiales; la ciencia produce conocimiento sólido y avanza rápido porque se basa en avances previos y combina observación cuidadosa con razonamiento riguroso para confirmar o rechazar ideas previas.

- La ciencia está sujeta a condicionamientos del entorno, incluyendo posturas ideológicas, y tiene implicaciones éticas y políticas.

Más allá de estas ideas básicas, una formación sólida en epistemología incluiría revisar a 
fondo las nociones de conocimiento y ciencia desde la filosofía griega, pasando por el empirismo de Bacon y Locke, la síntesis de Kant, el positivismo de Comte y el neopositivismo lógico del Círculo de Viena hasta llegar al post-positivismo de Popper, Kuhn y otros, la hermenéutica y la neo-hermenéutica de Gadamer, Betti o Apel, y la Escuela de Frankfurt de Habermas. También habría que estudiar el postmodernismo de Lyotard, Baudrillard o Foucault, el deconstruccionismo de Derrida, el anarquismo metodológico de Feyerabend, y el constructivismo radical de Von Glazersfeld y Watzlawick.

Enseñar es una tarea compleja; no es razonable exigir que, además de los contenidos a enseñar y del conocimiento pedagógico de los mismos, los docentes tengan conocimientos profundos de epistemología. Los textos de Phillips, 1995 y 2000; Phillips y Burbules, 2000; y Matthews, 2017 podrán ser punto de partida para saber más sobre constructivismo epistemológico.

Lo necesario es distinguir las posturas extremas sobre la ciencia y la intermedia que ofrece la epistemología actual, para aprovechar la solidez de las aportaciones del constructivismo en su primera acepción sobre aprendizaje y evitar los errores a que lleva la visión de una enseñanza sin orientación por parte del docente a la que puede llegar la segunda acepción del término.

\section{IMPLICACIONES DE LAS TRES}

\section{ACEPCIONES DEL CONSTRUCTIVISMO PARA LA DOCENCIA}

La idea de que toda teoría de la enseñanza supone cierta teoría del aprendizaje deja clara la relación entre las dos primeras acepciones del término constructivismo. La tercera acepción, epistemológica, se relaciona con las dos primeras así: si se considera que el conocimiento de sentido común o precientífico no es distinto del que producen las ciencias, puesto que ambos serían construcciones sociales, igualmente sujetas a condicionamientos sociales y culturales, entonces un docente no puede pretender que tiene conocimientos mejores que los de sus alumnos, ni pretender enseñarles algo; lo que debe hacer es dejarlos que descubran por sí mismos todos los conocimientos que puedan. El constructivismo epistemológico ofrece un argumento a favor de la versión extrema de enseñanza por descubrimiento no guiado.

Muchos docentes afirman que siguen el constructivismo, pero la observación en aula lleva con frecuencia a concluir que prevalecen prácticas tradicionales de enseñanza frontal, a veces con indicios de enseñanza no guiada. Las observaciones no sistemáticas del autor sobre la práctica de cientos de docentes que participaron en cursos de desarrollo profesional muestran que pocos aplican estrategias para desarrollar competencias de alto nivel, cognitivas y no cognitivas, pero también se vio que no todo es negativo: casi todos los docentes dominan estrategias básicas para manejo del aula y control de disciplina, o para aprendizajes básicos como leer textos sencillos, escribir recados o manejar las operaciones básicas de la aritmética. Hay pocos docentes cuyas prácticas se apegan a las ideas pedagógicas avanzadas, pero también pocos que presentan los peores rasgos de enseñanza frontal y autoritaria; la mayoría se esfuerza por atender a sus alumnos, con los límites que imponen las condiciones en que trabajan, las características de sus alumnos y su propia formación.

En México hay menos investigaciones empíricas sobre prácticas docentes que las deseables, pero las que hay, intensivas o extensivas, suelen señalar que en las aulas prevalece una enseñanza frontal, centrada en el docente, con pocos ejemplos de enseñanza que promueva la actividad intelectual del alumno, pero también encuentra prácticas positivas.

Con estudios etnográficos, Candela destaca el esfuerzo de algunos maestros por dialogar con sus alumnos y construir con ellos significados; en cursos comunitarios, con la 
propuesta “Dialogar y descubrir", observó formas de trabajo más cercanas a una enseñanza constructivista (Candela et al., 2012). Otros trabajos, de carácter extensivo, reportan la presencia dominante de prácticas rutinarias y memorísticas, como leer el libro de texto, subrayarlo o copiar pasajes (Flores-Camacho, 2012; Valdez, 2012).

En un estudio sobre enseñanza de ciencias se videograbaron 45 clases; se encontró que las prácticas se enfocan a "exposición de contenidos con definiciones y/o a contextualización que no deja espacio para interpretaciones por parte de los estudiantes" prácticas que "permiten interacciones centralizadas... alrededor de conocimientos científicos" (Rodríguez-Pineda y López-Mota, 2012: 177).

Un estudio de observación en 279 escuelas de Paraguay, República Dominicana y México (101 primarias de Nuevo León) incluyó grabar una clase de matemáticas y una de ciencias de $6^{\circ}$ de primaria en cada escuela; se encontró que:

Los docentes... dependen principalmente de la presentación y repetición de los procedimientos matemáticos... combinados con la repetición, práctica y memorización de conceptos... se dedican a copiar del pizarrón y a completar números en las fórmulas. Solamente en Nuevo León hemos identificado una proporción mayor de docentes que intentan salir de la simple repetición de los procedimientos y hacen participar activamente a sus alumnos en tareas que permiten dotarlos de destrezas de pensamiento crítico y analítico [pero] predominan la repetición mecánica, [la] práctica y [la] memorización de conceptos. Las presentaciones dirigidas por el docente ocupan la mayor parte del tiempo (Näslund-Hadley y Loera Varela, 2011: 2-3).

La combinación de aspectos positivos y negativos en las prácticas docentes está presente en los resultados de un estudio intensivo, según los cuales:
Prevalecen las consignas claras y rutinas con las que se organiza el trabajo con rapidez y se sostiene buen ritmo, pero hay mucho camino que recorrer para que las actividades escolares sean menos directivas, representen mayores retos cognitivos... se privilegia el trabajo individual por mantener el orden o por las dificultades provocadas por el espacio y mobiliario... el tiempo es escaso, hay interrupciones... los maestros casi siempre mantienen a los alumnos involucrados y trabajando... En general observamos un buen ambiente de aprendizaje... pocos episodios de discriminación, segregación o exclusión por parte de los compañeros... es notorio el compromiso que los maestros muestran en su afán de atender a todos los niños cuando trabajan con alumnos que presentan condiciones y características que desafían sus competencias docentes: rezago educativo y ausentismo... migración, violencia social e intrafamiliar... Hay avances en asumir la diversidad... pero hay dificultades... se baja el nivel de exigencia, se trabajan sólo contenidos básicos... ceremonias, guardias, reuniones de consejo técnico, cooperativa, rutas de mejora... quitan tiempo... Son pocos los momentos... para tener intercambios con sus pares... (Weiss et al., 2019: 355-357).

Personas ajenas a la escuela a veces afirman que nada ha cambiado en décadas, pero cuando algunos fuimos a primaria era normal que el maestro castigara la mala conducta con golpes, o hiciera ponerse de rodillas en un rincón, eventualmente con orejas de burro, a quien, a su juicio, lo merecía. Hoy el castigo corporal es excepcional, e incluso algunos padres incurren en excesos que ponen a la defensiva al docente. Un estudio serio apoya esta visión.

En la década de 1980 Elsie Rockwell observó el trabajo docente en siete escuelas de Tlaxcala; 30 años después, en las mismas escuelas encontró feminización de la docencia y la dirección; docentes y personal de apoyo además del titular; menos viajes por asuntos administrativos porque algunos los arregla 
un ATP o Internet, aunque aumenta la carga administrativa al deber continuamente subir informaciones; papel más activo del director; menor peso del sindicato; ya casi no hay mesabancos fijos y sí libros de la biblioteca de aula y equipos informáticos; alumnos que trabajan en grupo o exponen; menos ausentismo de docentes, pero frecuentes interrupciones por actividades internas; ritmos de trabajo más rápidos y menos tiempos muertos; docencia no limitada a español-matemáticas, tiempo para ciencias, historia, formación cívica y ética, educación artística y física, a veces con maestros especializados.

Rockwell observó que persisten limitaciones en estrategias didácticas, demasiado centradas en repetición y mecanización; las TIC no siempre funcionan y se usan superficialmente; los docentes se sienten abrumados por la velocidad de los cambios y la cantidad de información que se les pide asimilar. Una mirada superficial que advierta que sigue habiendo pizarrón y textos impresos, que predomina el maestro que interroga y evalúa, y alumnos que responden, puede concluir que las escuelas no han cambiado. Según la autora, sin embargo, una mirada más fina permite argumentar que el trabajo sí ha cambiado, y mucho:

El trabajo docente se ha transformado durante los últimos treinta años... de 24 docentes... encontré solo dos que ni querían estar frente a grupo, ni hacían gran cosa. En cambio, hubo fácil ocho maestros y maestras excepcionales, todos distintos, con una capacidad de convocar a los alumnos, a entregarse a actividades con sentido y a participar en discusiones que rebasaban con mucho lo esperado en los programas y libros. Más de la mitad sorteaban la situación y realizaban un trabajo digno, con una atención sensible hacia las necesidades de sus alumnos... había quienes cumplían medianamente, pero sobre todo quienes, recién iniciados en el oficio, batallaban para aprender sin mayor apoyo el secreto del manejo del grupo, intentando a la vez poner en práctica métodos novedosos y comunicarse en otros planos con los jóvenes a su cargo... Hace falta comprender y mejorar las condiciones del trabajo, considerando características de los espacios, las limitaciones de los tiempos, las formas de organizar grupos de alumnos y redes de docentes (Rockwell, 2013: 455-456, 470-472).

\section{Conclusión}

Lo expuesto lleva a concluir que no hay sustento para visiones extremas, positivas o negativas, de la práctica docente; más bien coexisten esfuerzos por atender a los alumnos, pero la enseñanza prevaleciente no desarrolla competencias complejas.

Esta visión matizada es congruente con una lectura objetiva de los resultados de las pruebas PISA, distinta de las alarmistas usuales. Es cierto que pocos jóvenes mexicanos (unos 5 de cada 100) alcanzan los niveles más altos que miden las pruebas, que indicarían que tienen competencia suficiente para ocupar puestos directivos o de investigación, desarrollo e innovación. Pero también lo es que más de la mitad (55 por ciento) de los chicos se ubica en niveles intermedios, lo que quiere decir que tienen las competencias mínimas que exige la sociedad del conocimiento. Además, la mayor parte del resto (unos 30 de cada 100) alcanza el nivel 1a de PISA, que significa que tienen competencias básicas, pero no suficientes para desenvolverse en sociedades avanzadas; una parte no desdeñable, pero menor (alrededor de 10 de cada 100) se encuentra todavía más abajo en la escala de competencia; y una minoría muy pequeña, de un 2 por ciento, no alcanza siquiera ese nivel mínimo (cfr. INEE, 2010; 2013; 2017).

Estos datos indican que los docentes consiguen que más de la mitad de sus alumnos alcancen niveles aceptables, pero pocos logran que una proporción importante llegue a los niveles altos. Estas visiones del vaso medio lleno o medio vacío se explican si se tiene presente lo difícil que es una práctica docente 
que lleve a todos los chicos, en especial a los de medio vulnerable, a desarrollar competencias complejas. Una mejora sustancial de los niveles de aprendizaje de los jóvenes de un sistema educativo tan grande y heterogéneo como el mexicano, además de bastante tiempo, supondrá implementar políticas eficaces que atemperen las desigualdades del hogar, y mejoren también la formación inicial de los docentes y la actualización que se les ofrece cuando ya están en servicio. Supondrá también humildad de investigadores y tomadores de decisiones para reconocer que no hay soluciones mágicas, y que hay que apoyar la docencia.

Al sintetizar hallazgos sobre cómo han enseñado los maestros desde fines del siglo XIX, Cuban distingue dos tradiciones que pueden remontarse hasta los griegos, una centrada en el maestro (mimética, de trasmisión, directa), y otra en los alumnos (progresiva, facilitadora, transformadora, constructivista). En la primera el aprendiz es descrito con metáforas como pizarra en blanco (tabula rasa) o recipiente a llenar; en la segunda se habla de descubrir o construir el aprendizaje, por alumnos que son "un jardín floreciente que necesita un jardinero competente" (Cuban, 2009: 6).

Los partidarios de ambas tradiciones creen que, independientemente de sus antecedentes, todos los alumnos pueden captar contenidos, adquirir habilidades, cultivar actitudes y desarrollar conductas, y que lo harán mejor gracias a prácticas de la tradición que defienden, pero las evidencias acumuladas sobre cómo producen resultados concretos entre los alumnos las prácticas reales en el aula son mezcladas y poco convincentes. Por lo tanto, aún no hay evidencia mayoritaria que demuestre la superioridad inherente de una u otra pedagogía... (Cuban, 2009: 6).

A falta de evidencia, las preferencias se basan en ideología y fe, pues la distinción entre las tradiciones se diluye, $y$ "los maestros han desarrollado cierta autonomía para arreglar el espacio y organizar actividades en el aula... en forma de rutinas prácticas y métodos de enseñanza de tradiciones distintas" (Cuban, 2009: 10).

Las prácticas de cada maestro, además, no son uniformes; tienen variantes según los niños y el grupo, contenidos, momento y contexto. El buen maestro se distingue por su capacidad para combinar estrategias de las dos tradiciones que, además:

No son dicotómicas; siempre ha habido híbridos... Mis investigaciones y experiencia mellevan a pensar que ninguna de estas tradiciones, defínase como se defina, es la mejor forma de enseñanza para todos los alumnos... Creo que los híbridos de ambas pedagogías -en sentido de que el repertorio de un maestro incluya múltiples enfoques, en función de diferencias del contexto, de quiénes son los estudiantes, del contenido a enseñar y de otras circunstancias- son los que tienen más probabilidades de conseguir que la mayoría de los alumnos aprendan (Cuban, 2009: nota 9, pp. 79-80).

El eclecticismo de los docentes experimentados parece, pues, una postura razonable. Las teorías del aprendizaje han dado lugar a estrategias pedagógicas tan variadas como ellas, y al parecer todas han dado resultados en algunas circunstancias. Como ejemplos se puede mencionar el aprendizaje de dominio, la enseñanza programada, la resolución de problemas, la enseñanza por competencias, los conflictos cognitivos, la modificabilidad cognitiva y el enriquecimiento instrumental, o las muchas propuestas especializadas en didáctica de la lectoescritura, de las matemáticas o de las ciencias naturales. Por otra parte, en otras circunstancias esas mismas propuestas han dado escasos resultados y son cuestionadas por quienes sostienen puntos de vista diferentes. El paso de la teoría a la práctica es crucial; la implementación de cualquier propuesta depende de muchas variables y condiciones del contexto, de suerte que se impone una actitud cautelosa. 
Las reformas suelen proponer uno $\mathrm{u}$ otro de dos extremos sobre cómo mejorar la enseñanza:

- Al desconfiar de la capacidad de los maestros para hacer una enseñanza de gran calidad, unos apuestan a un currículo y materiales de apoyo (libros de texto $y$ otros) tan detallados que cualquier docente, incluso poco capaz, pueda implementarlo: un currículo "a prueba de maestros".

- Otros requieren que cada maestro sea extraordinariamente competente, con amplios conocimientos de las asignaturas, los alumnos y la pedagogía, pues esperan que haya "un Leonardo en cada aula” (Bryk et al., 2010).

Ambas posturas son inoperantes: sin buenos maestros, ningún currículo conseguirá que los alumnos tengan altos niveles de aprendizaje, pero tampoco es posible que cada maestro sea perfecto. Hay que basarse en el trabajo de maestros no perfectos, pero responsables, que se superen continuamente trabajando

\section{REFERENCIAS}

Abell, Sandra K., Ken Appleton y Deborah L. Hanuscin (2010), Designing and Teaching the Elementary Science Methods Course, Nueva York, Routledge.

Alexander, Robin (2000), Culture and Pedagogy. International comparisons in primary education, Malden, Blackwell Publ.

BALL, Deborah L. y Heather C. Hill (2009), "Measuring Teacher Quality in Practice”, en Drew H. Gitomer (ed.), Measurement Issues and Assessment for Teaching Quality, Los Angeles, Sage, pp. 80-89.

Bruner, Jerome S. (1961), "The Art of Discovery", Harvard Education Review, vol. 31, pp. 21-32.

BRUNER, Jerome S. (1966), "Some Elements of Discovery”, en Lee S. Shulman y Evan R. Keislar (eds.), Learning by Discovery. A critical appraisal, Chicago, Rand McNally Co., pp. 101-113.

Bryk, Anthony S., Penny Bender Sebring, Elaine Allensworth, Stuart Luppescu y John Q. Easton (2010), Organizing Schools for Improvement. Lessons from Chicago, Chicago/Londres, The University of Chicago Press. con sus colegas, en comunidades profesionales de aprendizaje basadas en la escuela, lo que implica:

- Abrir el trabajo de cada maestro en el aula para que sea observado por colegas y asesores.

- Instalar un diálogo crítico sobre prácticas de aula: ¿qué ocurre?, ¿cómo saber si algo funciona?, ¿qué evidencia hay de que los alumnos están aprendiendo?, ¿hay otras prácticas que funcionen mejor?, ¿cómo podríamos saber cuáles?

- Una colaboración sostenida entre maestros, centrada en el fortalecimiento del sistema de enseñanza de la escuela (Bryk et al., 2010).

Muchas acciones de actualización han sido cortas, teóricas, alejadas del aula y dirigidas a individuos. Hay que promover los rasgos opuestos: larga duración; partir de la práctica; reflexionar sobre ella con elementos teóricos; trabajar cerca de aula y escuela; y dirigirse a colectivos, con el director o la directora al frente, en verdaderas comunidades de aprendizaje.

Candela, Antonia, Enna Carvajal, Armando Sánchez y Clara Alvarado (2012), "La investigación en las aulas de ciencias y la formación docente", en Fernando Flores Camacho (coord.), La enseñanza de la ciencia en la educación básica en México, México, INEE, pp. 33-56.

Clark, Richard E. (2009), "How Much and What Type of Guidance is Optimal for Learning from Instruction?", en Sigmund Tobias y Thomas M. Duffy (eds), Constructivist Instruction. Success or failure?, Nueva York/ Londres, Routledge, pp. 158-183.

Crahay, Marcel (2002), Psicología de la educación, Santiago de Chile, Ed. Andrés Bello.

Cuban, Larry (2009), Hugging the Middle. How teachers teach in an era of testing and accountability, Nueva York, Teachers College Press.

American Educational Research Association (2007), "Constructivism discovery, problem based, experiential and inquiry-based teaching: Success or failure?", The World of Educational 
Quality. AERA 2007 Annual Meeting Program, Washington, AERA, pp. 218-219.

Flores-Camacho, Fernando (2012), "Conocimientos, concepciones y formación de los profesores", en Fernando Flores Camacho (coord.), La enseñanza de la ciencia en la educación básica en México, México, INEE, pp. 113-128.

Guskey, Thomas R.T. (2007), "Formative Classroom Assessment and Benjamin S. Bloom: Theory, research and practice", en James H. McMillan (ed.), Formative Classroom Assessment: Theory into practice, Nueva York, Teachers College Press, pp. 63-78.

Instituto Nacional para la Evaluación de la Educación (2010), México en PISA 2009, México, INEE.

Instituto Nacional para la Evaluación de la Educación (2013), México en PISA 2012, México, INEE.

Instituto Nacional para la Evaluación de la Educación (2017), México en PISA 2015, México, INEE.

KintsCH, Walter (2009), "Learning and Constructivism", en Sigmund Tobias y Thomas M. Duffy (eds), Constructivist Instruction. Success or failure?, Nueva York/London, Routledge, pp. 223-241.

Kirschner, Paul A., John Sweller y Richard E. Clark (2006), "Why Minimal Guidance During Instruction Does Not Work: An analysis of the failure of constructivist, discovery, problem-based, experiential and inquirybased teaching", Educational Psychologist, vol. 41, núm. 2, pp. 75-96. DOI: https://doi. org/10.1207/s15326985ep4102_1

Knoers, A. (1994), "Instructional Psychology Paradigms", en Torsten Husén y Postlethwaite T.S. Neville (eds.), International Encyclopedia of Education, Oxford/Nueva York, Pergamon, vol. 5, pp. 2880-2884.

Matthews, Michael R. (2017), La enseñanza de la ciencia. Un enfoque desde la historia y la filosofía de la ciencia, México, Fondo de Cultura Económica.

MAyer, Richard E. (2004), "Should There Be a 3-Strike Rule Against Pure Discovery Learning? Case for guided methods of instruction", American Psychologist, vol. 59, núm. 1, pp. 14-19, en: http://tictrabalhodeprojecto.pbworks. com/f/Should\%20There\%20Be\%20a\%20 Three-Strikes\%20Rule\%20Against\%20Pure. pdf (consulta: 2 de octubre de 2020).

Mayer, Richard E. (2009), "Constructivism as a Theory of Learning Versus Constructivism as a Prescription for Instruction", en Sigmund Tobias y Thomas M. Duffy (eds), Constructivist Instruction. Success or failure?, Nueva York/Londres, Routledge, pp. 184-200.
Mayer, Richard E. (2014), "Cognitive Revolution and Information Processing Perspectives", en Denis C. Phillips (ed.), Encyclopedia of Educational Theory and Philosophy, vol. 1, Los Angeles, Sage, pp. 140-149.

NÄSLund-Hadley, Emma y Armando Loera Varela (2011), "El aula de matemáticas desde adentro: cualidades de un docente eficaz", BID Educación Aportes, núm. 11. https:// es.scribd.com/document/248676776/El-Aula-de-Matematicas-Desde-Adentro-Cualidades-de-Un-Docente-Eficaz (consulta: 2 de octubre de 2020).

Phillips, Denis C. (1995), "The Good, the Bad, and the Ugly: The many faces of constructivism", Educational Researcher, vol. 24, núm. 7, pp. 5-12. DOI: https://doi.org/10.3102/0013189 X024007005

Phillips, Denis C. (2000), Expanded Social Scientist's Bestiary: A guide to fabled threats to, and defenses of, naturalistic social science, Lanham, Rowman \& Littlefield Publ.

Phillips, Denis C. y Nicholas C. Burbules (2000), Postpositivism and Educational Research, Lanham, Rowman \& Littlefield Publ.

Rockwell, Elsie (2013), "El trabajo docente hoy: nuevas huellas, bardas y veredas", en COMIE, XI Congreso Nacional de Investigación Educativa. Conferencias magistrales, México, COMIE, pp. 437-473.

Rodríguez-Pineda, Diana P. y Ángel López-Mota (2012), "Description of Practice Teaching from the Systematic Observation of the Working Class in High School", en Catherine Bruguiére, André Tiberghien y Pierre Clément (eds.), E-book Proceedings of the ESERA 2011 Conference: Science learning and citizenship, Lyon, European Science Education Research Association, pp. 174-178.

Schunk, Dale H. (2014), "Learning, Theories of", en Denis C. Phillips (ed.), Encyclopedia of Educational Theory and Philosophy, vol. 2, Los Angeles, Sage, pp. 466-469.

Shulman, Lee S. (1986), “Those Who Understand: Knowledge growth in teaching”, Educational Researcher, vol. 15, núm. 2, pp. 4-14. DOI: https://doi.org/10.3102/0013189X015002004

Shulman, Lee S. (1990), "Aristotle Had It Right: On knowledge \& pedagogy. Holmes Group”, en Lee S. Shulman (2004), The Wisdom of Practice. Essays on teaching, learning, and learning to teach, San Francisco, Jossey Bass, pp. 400-415.

Shulman, Lee S. y Evan R. Keislar (eds.) (1966), Learning by Discovery. A critical appraisal, Chicago, Rand McNally Co., pp. 101-113.

Sommerfeld Gresalfi, Melissa y Frank Lester (2009), "What's Worth Knowing in Mathematics?", en Sigmund Tobias y Thomas M. Duffy (eds), Constructivist Instruction. Success or failure?, Nueva York/Londres, Routledge, pp. 264-290. 
Sweller, John (2009), "What Human Cognitive Architecture Tells Us About Constructivism", en Sigmund Tobias y Thomas M. Duffy (eds.), Constructivist Instruction. Success or failure?, Nueva York/Londres, Routledge, pp. 127-143.

Swenson, Leland C. (1980), Theories of Learning. Traditional perspectives/contemporary developments, Belmont, Wadsworth Publ. Co.

Tobias, Sigmund y Thomas M. Duffy (eds.) (2009), Constructivist Instruction. Success or failure?, Nueva York/Londres, Routledge.

VAldez González, Ricardo (2012), "Materiales educativos y recursos didácticos de apoyo para la educación en ciencias", en Fernando Flores Camacho (coord.), La enseñanza de la ciencia en la educación básica en México, México, INEE, pp. 93-112.

Weiss, Eduardo, David Block, Alicia Civera, Amira Dávalos y Gabriela Naranjo (2019), "La enseñanza de distintas asignaturas en escuelas primarias: una mirada a la práctica docente", Revista Mexicana de Investigación Educati$v a$, vol. 24, núm. 81, pp. 349-374, en: https:// www.comie.org. mx/revista/v2018/rmie/index.php/nrmie/article/view/1269/1198 (consulta: 2 de octubre de 2020). 